\title{
ON THE THICKNESS OF THE COMPLETE GRAPH ${ }^{1}$
}

\author{
BY LOWELL W. BEINEKE AND FRANK HARARY
}

Communicated by J. W. T. Youngs, January 22, 1964

The thickness $t\left(K_{p}\right)$ of the complete graph $K_{p}$ with $p$ points is the minimum number of planar subgraphs whose union is $K_{p}$. The purpose of this note is to outline a result which determines $t\left(K_{p}\right)$ for four of every six consecutive integers $p$. A complete proof of this result will be published elsewhere.

Theorem. If $p \equiv-1,0,1,2(\bmod 6)$, then

$$
t\left(K_{p}\right)=\left[\frac{p+7}{6}\right] .
$$

In proving this theorem, we prescribe a labelling of $n+1$ plane graphs, for any positive integer $n$. All the graphs contain the same $6 n+2$ points, but are constructed so that no two have a common line. Two of the points will be denoted by $v$ and $v^{\prime}$, and the others as $u_{k}, v_{k}, w_{k}, u_{k}^{\prime}, v_{k}^{\prime}, w_{k}^{\prime}$ for $k=0,1, \cdots, n-1$. All but one of the graphs are of the type indicated in Figure 1, where each of the six numbered triangles in $G_{k}$ contains $n-1$ other points and $3(n-1)$ lines so that its interior is isomorphic with graph $H$.

The points of the $n$ graphs $G_{k}$ are labelled using an $n \times n$ matrix $A=\left(a_{i j}\right)$, whose entries are residue classes modulo $n$, where

$$
a_{i j}=\left((-1)^{i}\left[\frac{i}{2}\right]+(-1)^{j}\left[\frac{j}{2}\right]\right)(\bmod n)
$$

with $[x]$ indicating the greatest integer function as usual. We remark that one of the important properties of $A$ is that each residue class appears exactly once in each row and each column.

The $n-1$ points inside triangle $u_{k}^{\prime} v_{k} w_{k}^{\prime}$ of graph $G_{k}$ are labelled using the column, say the $j$ th, whose first entry is $a_{1 j}=k$ as follows: if $a_{i j}=h$, the $(i-1)$ st point down from $v_{k}$ is labelled $v_{h}$ or $v_{h}^{\prime}$ according as $\min \{i, j\}$ is odd or even. The points inside triangle $v_{k} u_{k}^{\prime} w_{k}$ are similarly labelled, using $u_{h}^{\prime}$ and $u_{h}$ instead of $v_{h}$ and $v_{h}^{\prime}$ respectively. The points inside the other triangles are also labelled analogously.

Now, in the union of these $n$ labelled graphs $G_{k}$, aside from $v$ and $v^{\prime}$, each point is adjacent with all but one of the other points. More-

\footnotetext{
1 This research was supported by National Science Foundation grant GP-207.
} 

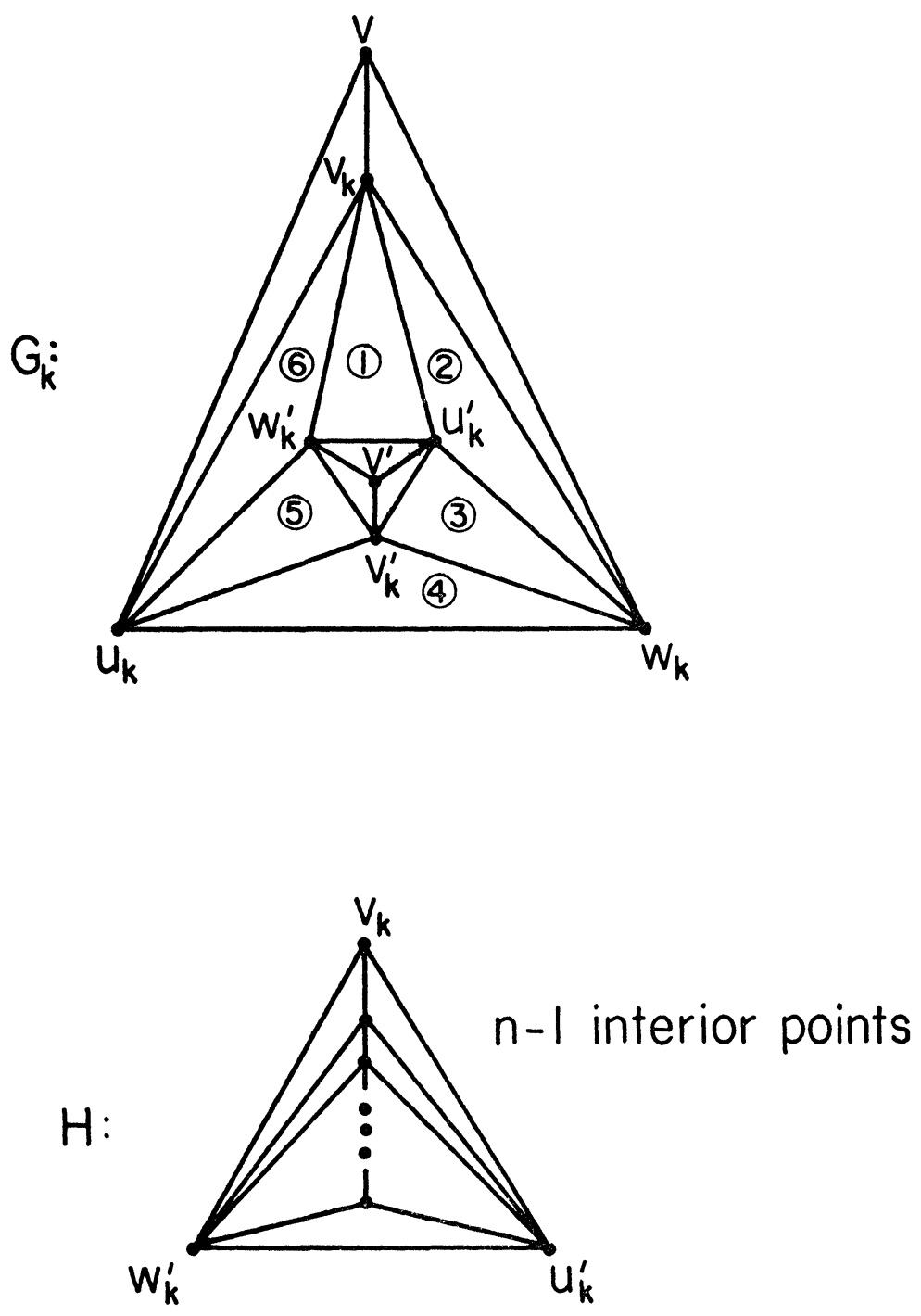

FIGURE 1

over, for each integer $k$, the points $u_{k}, v_{k}$, and $w_{k}$ are not adjacent to $u_{k}^{\prime}, v_{k}^{\prime}$, and $w_{k}^{\prime}$, respectively. Also, $v$ and $v^{\prime}$ are each adjacent with half of the other points. A new graph $G$ is constructed as in Figure 2, in which each of the $6 n+2$ points is adjacent to all of the points not adjacent to it in any of the other $n$ graphs. Therefore the union of 


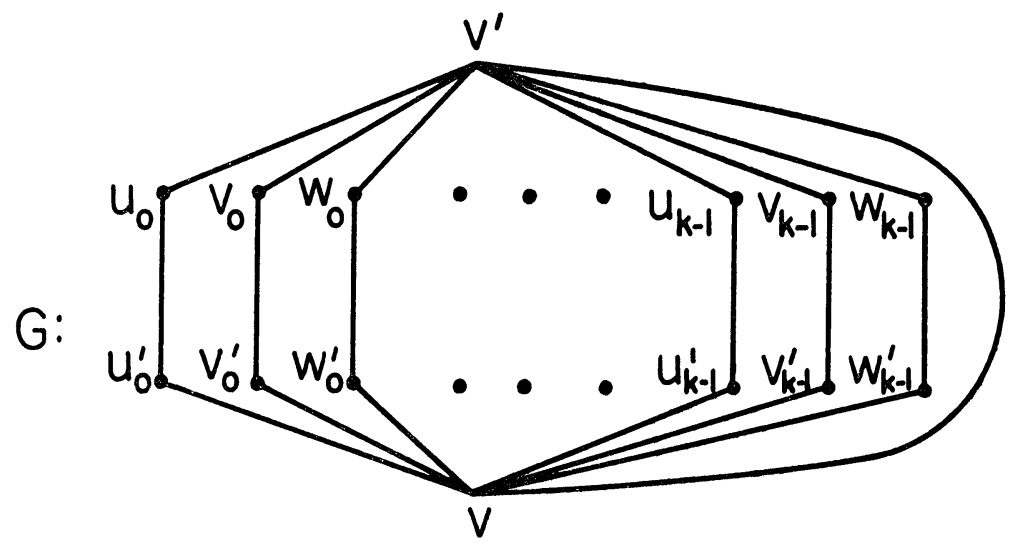

Figure 2

the graph $G$ with the $n$ graphs $G_{k}$ is complete. Thus, $t\left(K_{6 n+2}\right) \leqq n+1$. From Euler's polyhedron formula it follows that $t\left(K_{6 n-1}\right) \geqq n+1$. The theorem follows at once from these two inequalities.

UNIVERSITY OF MichigAN AND

UNIVERSITY COLLEGE, LONDON, ENGLAND 